\title{
Playing tennis matches on clay court surfaces are associated with more perceived enjoyment response but less perceived exertion compared to hard courts
}

\author{
Bulent Kilit ${ }^{1}$ and Ersan Arslan ${ }^{2, *}$ \\ ${ }^{1}$ School of Physical Education and Sports, Namik Kemal University, Tekirdag, Turkey; and ${ }^{2}$ School of Physical Education \\ and Sports, Siirt University, Siirt, Turkey
}

Copyright: (C) 2018 B. Kilit and E. Arslan. This is an open access article licensed under the Creative Commons Attribution License (http://creativecommons.org/licenses/by/4.0/).

\begin{abstract}
Background: A few studies have shown that different types of court surfaces can have different effects on tennis match performance in different age groups, however, no studies have researched on tennis match performance responses in young tennis players. Objective: The purpose of this study was to evaluate the effect of different court surfaces on psychophysiological responses, time-motion and match characteristics of young tennis players. Methods: Twenty-six young male tennis players (age $13.0 \pm 0.3$ years, body height $158 \pm 8 \mathrm{~cm}$, body mass $48 \pm 7 \mathrm{~kg}$ ) volunteered to participate in the study. Heart rate was monitored along with total distance covered in four different speed zoneswalking, low-intensity running, moderate-intensity running and high-intensity running, using two portable multivariable integrated $10 \mathrm{~Hz}$ GPS monitoring devices over twenty-six outdoor tennis matches; ratings of perceived exertion and enjoyment level were also determined at the end of the matches. Variables describing the characteristics of the matches determined from video recordings were: strokes per rally, rally duration, effective playing time, work-to-rest ratio and rest time between rallies. Differences between hard and clay courts were assessed by a paired t-test. Results: The results showed that clay court surfaces induced significantly more perceived enjoyment scores $(29.4 \pm 2.8$ vs. $26.0 \pm 3.2)$ with lower perceived exertion level $(13.4 \pm 2.7$ vs. $14.4 \pm 2.9)$ compared to hard courts. Moreover, a clay court surface is also more effective at improving physiological responses, time-motion and match characteristics compared to hard court. Conclusions: If a coach strives to improve their young tennis players' tennis-specific performance with greater physical enjoyment, clay court surfaces should be specially used in their tennis training season.
\end{abstract}

Keywords: performance profile, multivariable monitoring, GPS, court surfaces, psychophysiological responses

\section{Introduction}

Effective performance in tennis requires running at different speeds, acceleration, deceleration, turns, changeovers, strokes, sprints, sliding and upper arm involvement. It is an anaerobic sport with aerobic breaks between the rallies, making players perform short bursts of high-intensity exercise interspersed with periods of rest or low-intensity activities over a prolonged period (Fernandez-Fernandez, Mendez-Villanueva, \& Pluim, 2006; Reid et al., 2013). A recent study has focused on different performance indicators in tennis. Some of them were time-motion characteristics (Galé-Ansodi,

\footnotetext{
* Address for correspondence: Ersan Arslan, Department of Coaching Education, School of Physical Education and Sports, Siirt University, Kezer Yerleșkesi MuhendislikMimarlik Fakultesi B Blok, 56100 Siirt, Turkey. E-mail: ersanarslan1980@hotmail.com
}

Castellano, Usabiaga, \& 2016; Hoppe et al., 2014; Kilit $\&$ Arslan, 2017; Pereira et al., 2016). In this regard, previous studies of simulated tennis matches have shown that young tennis players cover a distance of $2.7-3.4 \mathrm{~km}$ with an average 160 heart rate (Hoppe et al., 2014; Kilit, Şenel, Arslan, \& Can, 2016; Reid et al., 2013). In addition to these factors, it is well known from research on simulated matches that match characteristics were affected by not only different playing conditions (Kilit \& Arslan, 2018; Kilit et al., 2016; Tsetseli, Zetou, Vernadakis, \& Michalopoulou, 2016), but also by different court surfaces (Martin et al., 2011).

Court surfaces are classified into five categories: slow, medium-slow, medium, medium-fast, and fast according to the International Tennis Federation (2018). The three court surfaces, which are clay, hard and grass are used to playing multiple tournaments in professional and recreational tournament calendars. Many previous studies of simulated tennis matches 
have shown that time-motion characteristics (GaléAnsodi et al., 2016; Murias, Lanatta, Arcuri, \& Laino, 2007; Pereira et al., 2016; Ponzano \& Gollin, 2017), psychophysiological (Chapelle, Clarys, Meulemans, \& Aerenhouts, 2017; Fernandez-Fernandez, Kinner, \& Ferrauti, 2010; Girard \& Millet, 2004; Reid et al., 2013) and match characteristics (Girard \& Millet, 2004; Martin et al., 2011; O’Donoghue \& Ingram, 2001) were influenced by different court surfaces which are clay and hard. With the exception of two recent studies that used a large sample of subjects (Chapelle et al., 2017; Ponzano \& Gollin, 2017), the majority of studies have used small samples that ranged from 4 to 14 subjects. Moreover, findings of these studies are not uniform. For example, a previous study showed no significant difference in physiological responses between clay and hard courts in competitive tennis players (Hornery, Farrow, Mujika, \& Young, 2007). However, Martin et al. (2011) have recently showed significantly higher physiological responses on clay courts compared to hard courts.

Although many previous studies had investigated psychophysiological responses, time-motion and match characteristics across different match duration, gender and performance levels, no study has investigated the effects of different court surfaces on these performance variables. Thus, the purpose of this study was to assess the influence of different court surfaces on psychophysiological responses, time-motion and match characteristics of young tennis players.

\section{Methods}

\section{Participants}

Twenty-six young male tennis players (age $13.0 \pm 0.3$ years, body height $158 \pm 8 \mathrm{~cm}$, body mass $48 \pm 7$ $\mathrm{kg}$ ) volunteered to participate in the study. At the time of the study, the players involved trained 15-20 hours per week and had International Tennis Numbers ranging from 4 to 6 . All of the participants were right-handed tennis players. The players were already familiar with all the tests. All the players and their parents were notified of the research procedures, requirements, benefits and potential risks before providing written informed consent. This study was approved by the Research Ethics Committee of the Samsun Ondokuz Mayis University (B.30 2.ODM.0.20.08/311-448/15.08.2016) and was conducted in a manner consistent with the institutional ethical requirements for human experimentation in accordance with the Declaration of Helsinki.

\section{Experimental design}

A randomized cross-over design was used in order to examine the influence of different court surfaces on psychophysiological responses, time-motion and match characteristics in young tennis players. This study was performed over two weeks and included two main experimental sessions: on-court assessment; simulated singles tennis match play, during which players were monitored using portable integrated $10 \mathrm{~Hz}$ GPS multivariable devices, and match notational analysis. All matches took place at a similar time of day. Similar temperatures $\left(15-20{ }^{\circ} \mathrm{C}\right)$ and relative air humidity levels (40-45\%) were maintained throughout the study. All matches were performed on an outdoor hard and clay court surfaces.

\section{Instruments and procedure}

The International Tennis Number (ITN) test is an objective on-court assessment tool based on a set number of tennis-specific tasks and was developed to enable players to benchmark their tennis level against themselves and others worldwide (International Tennis Federation, 2004). The ITN test was performed according to the instructions provided by the International Tennis Federation (ITF). A ball machine (Tennis Tutor Plus, Sports Tutor Inc., Burbank, CA, USA) was used to feed balls to the tested players. The Hit and Turn Tennis test (HTTT), an acoustically controlled progressive on-court fitness test for tennis players, was performed according to the procedures suggested by Ferrauti, Kinner, and Fernandez-Fernandez (2011). The highest heart rate (HR) measurement during the test was recorded as $\mathrm{HR}_{\text {max }}$. After the test, estimated $\mathrm{VO}_{2 \max }$ was calculated for boys under 14 years old, by using the following formula (Ferrauti et al., 2011): $\mathrm{VO}_{2 \max }=30.0+1.66 \times($ player finishes level in HTTT $)$.

Prior to each match, all players performed a standardized 10-min warm-up, including forehand and backhand shots, volleys, overhead shots and serves, integrating game-specific actions. Each subject completed a total of a randomized a simulated tennis match on each outdoor court surfaces (hard and clay). All the twenty-six matches were played in random order according to the rules of the ITF (the best of three sets; Ojala \& Häkkinen, 2013). A set of three new balls (Wilson US Open, Chicago, IL, USA) was used for each match. A portable reliable and valid multivariable monitoring devices (Bioharness 3, GPS Sports Systems., Annapolis, MD, USA) with integrated $10 \mathrm{~Hz}$ GPS units (BT-Q818XT, Qstarz International, Taipei, Taiwan; Johnstone, Ford, Hughes, Watson, \& Garrett, 2012; Johnstone, Ford, Hughes, Watson, Mitchell, \& Garrett, 2012; Kilit \& Arslan, 2017) were used to 
record each player's physiological responses (heart rate) and time-motion characteristics (speed and total distance covered in different speed zones) during the matches. The players were familiar with the use of these devices in training sessions. The players wore a special harness that enabled these devices to be fitted to the upper part of their chest. Our subjects were fully familiarised with the 6-20 RPE scale in our training sessions. After the matches, the data were downloaded to a computer and analyzed using the software package OmniSense Analysis (Version 4,0; Zephyr Technology, Annapolis, MD, USA). In line with previous studies, for data analysis purposes, four speed zones were selected: walking (W, $0-7.0 \mathrm{~km} \cdot \mathrm{h}^{-1}$ ), low-intensity running (LIR, 7.01-12.0 $\mathrm{km} \cdot \mathrm{h}^{-1}$ ), moderate-intensity running (MIR, $12.01-18.0 \mathrm{~km} \cdot \mathrm{h}^{-1}$ ) and high-intensity running (HIR, 18.01-24.00 $\mathrm{km} \cdot \mathrm{h}^{-1}$; Kilit \& Arslan, 2017; Pereira et al., 2016).

The play of each participant was recorded using two video cameras (60 frames per second; Sony HDRCX240 Full HD, Sony, Tokyo, Japan) positioned $2 \mathrm{~m}$ from the side of the court at the level of the service line and approximately $6 \mathrm{~m}$ above the court for the duration of the match (Fernandez-Fernandez, Sanz-Rivas, Fernandez-Garcia, \& Mendez-Villanueva, 2008). A specialized tennis analysis program (Kinovea, Version 0.8.15; www.kinovea.org) was used for the analysis of the matches, and the analysis of all of the matches was performed by the same experienced researcher (Fernandez-Fernandez, Mendez-Villanueva, Fernandez-Garcia, \& Terrados, 2007; Murias et al., 2007). On the basis of match data, the following variables were calculated for each game: the duration of the rallies in seconds (DR); the rest time in seconds, not including changes of ends (RT); the work-to-rest ratio, not including changes of ends (W:R); effective playing time (EPT; expressed as a percentage of the total time when the ball was in play during a game); and strokes per rally (SPR; shots). Ratings of perceived exertion (RPE; 6-20) were also determined at the end of the game. This scale has previously been used as an indicator of intensity in tennis (Kilit \& Arslan, 2017). Enjoyment of physical activity was measured using the short form of the physical activity enjoyment scale (PACES) after each match (Paxton et al., 2008). This scale, which includes 5 items scored on a 1-7 Likert scale, has been validated as an indicator of enjoyment level in training in young Turkish players (Mirzeoğlu \& Çoknaz, 2014).

\section{Statistical analyses}

Data were reported as means and standard deviations. Before using parametric tests, the assumption of normality was confirmed using the Kolmogorov-Smirnov test (with Lilliefors correction). A paired $t$-test was performed on each dependent variable, including psychophysiological responses, time-motion and match characteristics in order to compare hard vs. clay courts during simulated tennis match play. All statistical analyses were performed using IBM SPSS Statistics (Version 22; IBM, Armonk, NY, USA), and the level of statistical significance was established at $\alpha=.05$. Effect sizes (Cohen's $d$ ) were calculated for each dependent variable. The effect size statistics was considered as follows: 0.2 - trivial, 0.6 - small, 1.2 - moderate, 2.0 - large, and > 2.0 - very large (Hopkins, Marshall, Batterham, \& Hanin, 2009).

\section{Results}

Table 1 shows the psychophysiological responses, timemotion and match characteristics of young tennis players during the tennis matches played in different court surfaces. This table also demonstrates that clay court surfaces induced significantly higher psychophysiological responses such as HR and PACES. Conversely, RPE responses in hard court surface were significantly higher than clay court surface.

In addition, clay court surfaces showed significantly higher time-motion characteristics in terms of average speed (AS), total distance (TD), W, LIR, MIR and HIR. Moreover, clay court surface induced significantly higher match characteristics such as SPR, DR, EPT except for W:R and RT.

\section{Discussion}

The purpose of this study was to examine the influence of different court surfaces on psychophysiological responses, time-motion and match characteristics of young tennis players. Our results demonstrated that playing tennis matches on clay court surface induced more perceived enjoyment response, but less perceived exertion compared to hard court. Moreover, clay court surface is effective at improving time-motion and match characteristics.

Numerous recent studies have shown that psychophysiological responses (Chapelle et al., 2017; Fernandez-Fernandez et al., 2010; Martin et al., 2011; Reid et al., 2013) and time-motion characteristics (GaléAnsodi et al., 2016; Kilit \& Arslan, 2017; Ponzano $\&$ Gollin, 2017) were affected by not only different playing conditions (Kilit \& Arslan, 2018; Kilit et al., 2016), but also different court surfaces (Martin et al., 2011). In addition, all these variables have been found to affect the match characteristics (Kovalchik \& Ingram, 2018; Reid et al., 2013). Psychophysiological 
Table 1

Simulated tennis matches analysis

\begin{tabular}{lcccccc}
\hline & Hard court & Clay court & $t$ & $p$ & 95\% CI & Cohen's $d$ \\
\hline HR (beat $\cdot \mathrm{min}^{-1}$ ) & $160 \pm 18$ & $166 \pm 18$ & -4.91 & .001 & {$[-5.74,-2.46]$} & 0.33 \\
RPE & $14.4 \pm 2.9$ & $13.4 \pm 2.7$ & -3.01 & .001 & {$[-2.10,-1.21]$} & 0.36 \\
PACES & $26.0 \pm 3.2$ & $29.4 \pm 2.8$ & -3.41 & .001 & {$[-5.09,-2.86]$} & 1.13 \\
AS (km $\cdot \mathrm{h}^{-1}$ ) & $2.26 \pm 1.9$ & $2.58 \pm 2.1$ & -3.25 & .001 & {$[-0.51,-0.12]$} & 0.15 \\
TD (m) & $2733 \pm 390$ & $3201 \pm 398$ & -5.97 & $<.001$ & {$[-630.7,-305.5]$} & 1.18 \\
W (m) & $2415 \pm 389$ & $2855 \pm 384$ & -3.80 & .001 & {$[-684.6,-195.5]$} & 1.13 \\
LIR (m) & $205 \pm 60$ & $287 \pm 66$ & -4.34 & .001 & {$[-121.6,-41.8]$} & 1.29 \\
MIR (m) & $46 \pm 20$ & $71 \pm 16$ & -5.06 & $<.001$ & {$[-43.6,-17.8]$} & 1.38 \\
HIR (m) & $2.7 \pm 1.3$ & $4.9 \pm 1.8$ & -3.95 & .001 & {$[-3.34,-1.00]$} & 1.40 \\
SPR (shots) & $5.2 \pm 4.1$ & $7.1 \pm 3.9$ & -2.04 & $<.001$ & {$[-3.70,0.02]$} & 0.47 \\
DR (s) & $7.7 \pm 7.1$ & $12.0 \pm 9.2$ & -2.63 & .012 & {$[-7.47,-0.98]$} & 0.52 \\
EPT (\%) & $21.8 \pm 4.1$ & $26.7 \pm 4.4$ & -9.16 & $<.001$ & {$[-4.79,-3.06]$} & 1.15 \\
W:R & $0.27 \pm 0.80$ & $0.48 \pm 0.60$ & 7.01 & $<.001$ & {$[0.33,0.59]$} & 2.26 \\
RT (s) & $28.9 \pm 25.1$ & $25.8 \pm 26.2$ & 2.50 & .016 & {$[0.54,5.10]$} & 0.12 \\
\hline
\end{tabular}

Note. $\mathrm{CI}=$ confidence interval; $\mathrm{HR}=$ heart rate; $\mathrm{RPE}=$ rating of perceived exertion; PACES = physical activity enjoyment scale; AS = average speed; TD = total distance; $\mathrm{W}=$ walking; LIR = low-intensity running; MIR = moderate-intensity running; HIR = highintensity running; $\mathrm{SPR}=$ strokes per rally; $\mathrm{DR}=$ rally duration; $\mathrm{EPT}=$ effective playing time; $\mathrm{W}: \mathrm{R}=$ work-to-rest ratio; $\mathrm{RT}=$ rest time.

responses were determined by the HR, RPE and PACES in present study. To our knowledge, this study is the first to examine these variables in detail in youth tennis players during hard and clay court tennis matches. Our results showed that playing tennis on clay court surfaces induce higher psychophysiological responses such as HR and PACES with lower RPE compared with hard court surface. This study's results are in accordance with many studies which affirm that playing tennis on clay court surface improve physiological responses such as HR and RPE (Martin et al., 2011; Murias et al., 2007; Reid et al., 2013). In a previous study it was stated that no single variable strongly explained tennis performance (Kovacs, 2007). Therefore, an evaluation of the match performance should be taken into considering the importance of all affecting factors such as psychophysiological responses and match characteristics. The high number of strokes, short- and high-intensity activities (especially in serve games and clay court condition) cause more physical strain (Fernandez-Fernandez et al., 2007; MendezVillanueva, Fernandez-Fernández, Bishop, \& Fernandez-Garcia, 2010). Thus, more rallies on clay court games might explain with higher psychophysiological responses of tennis players to have a closer relationship with match characteristics in terms of the higher stroke per rally and the longer duration of rally (Kilit \& Arslan, 2017; Kilit et al., 2016; Mendez-Villanueva et al., 2010).
Another important finding of the match characteristics is that the effective playing time and work to rest ratio, these match characteristics might depend on the court surfaces condition. The present study is in agreement with the Martin et al. (2011) reported that higher effective playing time on clay court condition contrast to hard court surface. In addition, our findings are similar with the Murias et al. (2007) who presented higher work and rest ratio on clay court condition. These similar findings might be explained by the court surface characteristics. Clay court surfaces lead to a slower game and shot rhythm with higher physical enjoyment which include longer rallies and shorter rest time and also the players had more time to hit the ball or ready to play than hard court.

Nowadays, the development of technological tools such as wearable and multivariable monitoring devices including GPS features offers a highly practical way of monitoring match activity profile of players such as distances covered, and distance covered at different speeds, acceleration and deceleration during tennis training and matches (Galé-Ansodi et al., 2017; Hoppe et al., 2014; Kilit \& Arslan, 2017, 2018). However, few studies have focused on time-motion characteristics according to different court surfaces (hard vs. clay courts) in young tennis players (Galé-Ansodi et al., 2016; Pereira et al., 2016). The present study results demonstrated that time-motion characteristics (average speed and total distance covered in different speed zones) in clay court were higher compared to 
hard court results. Some studies found that the court surfaces had an effect on the total distances covered; tennis players showed higher total distances covered in clay court surface compared to hard court (Murias et al., 2007; Pereira et al., 2016). However, unlike our study result, Galé-Ansodi et al. (2016) showed that the total distances covered was lower on clay court than on hard court surfaces. These conflicting findings may be partly explained by the different methodology, age, sex and level of the participants. It might be also explained by match characteristics in different surfaces conditions.

There are several limitations of our study that should be taken into consideration before final conclusions are drawn. Firstly, our results might not be generalized for different age and sex group players. Another limitation are the lack of grass court and indoor court conditions. The last limitation is GPS devices are not providing reliable measures during high-intensity and intermittent exercise (Coutts \& Duffield, 2010; Duffield, Reid, Baker, \& Spratford, 2010). A major strength of this study is large sample size.

\section{Conclusions}

The present study's findings demonstrate that in young male players a clay court surface is more effective compared to a hard court for improving physiological responses, time-motion and match characteristics. In addition, playing tennis matches on clay court surfaces also induced more perceived enjoyment response but less perceived exertion compared to hard courts. These findings might be used for the different types of training adaptation in young tennis players during the season.

\section{Conflict of interest}

There were no conflicts of interest.

\section{References}

Chapelle, L., Clarys, P., Meulemans, S., \& Aerenhouts, D. (2017). Physiological responses, hitting accuracy and step count of a tennis drill in function of court surface: A randomized cross-over design. International Journal of Performance Analysis in Sport, 17, 622-629.

Coutts, A. J., \& Duffield, R. (2010). Validity and reliability of GPS devices for measuring movement demands of team sports. Journal of Science and Medicine in Sport, 13, 133-135.

Duffield, R., Reid, M., Baker, J., \& Spratford, W. (2010). Accuracy and reliability of GPS devices for measurement of movement patterns in confined spaces for court-based sports. Journal of Science and Medicine in Sport, 13, 523-525.

Fernandez-Fernandez, J., Kinner, V., \& Ferrauti, A. (2010). The physiological demands of hitting and running in tennis on different surfaces. Journal of Strength and Conditioning Research, 24, 3255-3264.

Fernandez-Fernandez, J., Mendez-Villanueva, A., Fernandez-Garcia, B., \& Terrados, N. (2007). Match activity and physiological responses during a junior female singles tennis tournament. British Journal of Sports Medicine, 41, 711-716.

Fernandez-Fernandez, J., Mendez-Villanueva, A., \& Pluim, B. M. (2006). Intensity of tennis match play. British Journal of Sports Medicine, 40, 387-391.

Fernandez-Fernandez, J., Sanz-Rivas, D., Fernandez-Garcia, B., \& Mendez-Villanueva, A. (2008). Match activity and physiological load during a clay-court tennis tournament in elite female players. Journal of Sports Sciences, 26, 1589-1595.

Ferrauti, A., Kinner, V., \& Fernandez-Fernandez, J. (2011). The Hit \& Turn Tennis Test: An acoustically controlled endurance test for tennis players. Journal of Sports Sciences, 29, 485-494.

Galé-Ansodi, C., Castellano, J., \& Usabiaga, O. (2016). Effects of different surfaces in time-motion characteristics in youth elite tennis players. International Journal of Performance Analysis in Sport, 16, 860-870.

Galé-Ansodi, C., Castellano, J., \& Usabiaga, O. (2017). More acceleration and less speed to assess physical demands in female young tennis players. International Journal of Performance Analysis in Sport, 17, 872-884.

Girard, O., \& Millet, G. P. (2004). Effects of the ground surface on the physiological and technical responses in young tennis players. In A. Lees, J.-F. Kahn, \& I. W. Maynard (Eds.), Science and Racket Sports III (pp. 43-48). London, United Kingdom: Routledge.

Hopkins, W., Marshall, S., Batterham, A., \& Hanin, J. (2009). Progressive statistics for studies in sports medicine and exercise science. Medicine \& Science in Sports \& Exercise, 41, 3-12.

Hoppe, M. W., Baumgart, C., Bornefeld, J., Sperlich, B., Freiwald, J., \& Holmberg, H. C. (2014). Running activity profile of adolescent tennis players during match play. Pediatric Exercise Science, 26, 281-290.

Hornery, D. J., Farrow, D., Mujika, I., \& Young, W. (2007). An integrated physiological and performance profile of professional tennis. British Journal of Sports Medicine, 41, 531-536.

International Tennis Federation. (2018). ITF rules of tennis. Retrieved from https://www.itftennis.com/ media/277603/277603.pdf

International Tennis Federation. (2004). International Tennis Number - testing procedure. Retrieved from http://www. tennisplayandstay.com/media/131803/131803.pdf

Johnstone, J. A., Ford, P. A., Hughes, G., Watson, T., \& Garrett, A. T. (2012). Bioharness ${ }^{\mathrm{TM}}$ multivariable monitoring device. Part II: Reliability. Journal of Sports Science and Medicine, 11, 409-417.

Johnstone, J. A., Ford, P. A., Hughes, G., Watson, T., Mitchell, A. C., \& Garrett, A. T. (2012). Field based reliability 
and validity of the Bioharness ${ }^{\mathrm{TM}}$ multivariable monitoring device. Journal of Sports Science and Medicine, 11, 643-652.

Kilit, B., \& Arslan, E. (2017). Physiological responses and time-motion characteristics of young tennis players: Comparison of serve vs. return games and winners vs. losers matches. International Journal of Performance Analysis in Sport, 17, 684-694.

Kilit, B., \& Arslan, E. (2018). Effects of high-intensity interval training vs. on-court tennis training in young tennis players. Journal of Strength and Conditioning Research. Advance online publication. doi:10.1519/JSC.0000000000002766

Kilit, B., Şenel, Ö., Arslan, E., \& Can, S. (2016). Physiological responses and match characteristics in professional tennis players during a one-hour simulated tennis match. Journal of Human Kinetics, 51, 83-92.

Kovacs, M. S. (2007). Tennis physiology. Sports Medicine, 37, 189-198.

Kovalchik, S. A., \& Ingram, M. (2018). Estimating the duration of professional tennis matches for varying formats. Journal of Quantitative Analysis in Sports, 14, 13-23.

Martin, C., Thevenet, D., Zouhal, H., Mornet, Y., Delès, R., Crestel, T., ... Prioux, J. (2011). Effects of playing surface (hard and clay courts) on heart rate and blood lactate during tennis matches played by high-level players. Journal of Strength and Conditioning Research, 25, 163-170.

Mendez-Villanueva, A., Fernandez-Fernández, J., Bishop, D., \& Fernandez-Garcia, B. (2010). Ratings of perceived exertion-lactate association during actual singles tennis match play. Journal of Strength and Conditioning Research, 24, 165-170.

Mirzeoğlu, A. D., \& Çoknaz, D. (2014). A validity and reliability study of physical activity enjoyment scale-short form for Turkish children and youth. International Journal of Human Sciences, 11, 672-687.
Murias, J. M., Lanatta, D., Arcuri, C. R., \& Laino, F. A. (2007). Metabolic and functional responses playing tennis on different surfaces. Journal of Strength and Conditioning Research, 21, 112-117.

O'Donoghue, P., \& Ingram, B. (2001). A notational analysis of elite tennis strategy. Journal of Sports Sciences, 19, 107-115.

Ojala, T., \& Häkkinen, K. (2013). Effects of the tennis tournament on players' physical performance, hormonal responses, muscle damage and recovery. Journal of Sports Science and Medicine, 12, 240-248.

Paxton, R. J., Nigg, C., Motl, R. W., Yamashita, M., Chung, R., Battista, J., \& Chang, J. (2008). Physical Activity Enjoyment Scale short form - does it fit for children? Research Quarterly for Exercise and Sport, 79, 423-427.

Pereira, T. J. C., Nakamura, F. Y., Jesus, M. T. D., Vieira, C. L. R., Misuta, M. S., Barros, R. M. L. D., \& Moura, F. A. (2016). Analysis of the distances covered and technical actions performed by professional tennis players during official matches. Journal of Sports Sciences, 35, 361-368.

Ponzano, M., \& Gollin, M. (2017). Movement analysis and metabolic profile of tennis match play: Comparison between hard courts and clay courts. International Journal of Performance Analysis in Sport, 17, 220-231.

Reid, M., Dufeld, R., Minett, G. M., Sibte, N., Murphy, A. P., \& Baker, J. (2013). Physiological, perceptual, and technical responses to on-court tennis training on hard and clay courts. Journal of Strength and Conditioning Research, 27, 1487-1495.

Tsetseli, M., Zetou, E., Vernadakis, N., \& Michalopoulou, M. (2016). The effect of internal and external focus of attention on game performance in tennis. Acta Gymnica, $46,162-173$ 